TRANSACTIONS OF THE

AMERICAN MATHEMATICAL SOCIETY

Volume 357, Number 7, Pages 2613-2625

S 0002-9947(05)03738-4

Article electronically published on March 1, 2005

\title{
THE BERGMAN METRIC AND THE PLURICOMPLEX GREEN FUNCTION
}

\author{
ZBIGNIEW BŁOCKI
}

\begin{abstract}
We improve a lower bound for the Bergman distance in smooth pseudoconvex domains due to Diederich and Ohsawa. As the main tool we use the pluricomplex Green function and an $L^{2}$-estimate for the $\bar{\partial}$-operator of Donnelly and Fefferman.
\end{abstract}

\section{INTRODUCTION}

Diederich and Ohsawa [14] have shown that if $\Omega$ is a smooth bounded pseudoconvex domain in $\mathbb{C}^{n}$, then the following lower bound for the Bergman distance in $\Omega$ holds: for a fixed $w_{0} \in \Omega$ and $w \in \Omega$ close to the boundary, one has

$$
\operatorname{dist}_{\Omega}\left(w, w_{0}\right) \geq \frac{1}{C} \log \log \left(1 / \delta_{\Omega}(w)\right),
$$

where $\delta_{\Omega}(w)$ denotes the euclidean distance of $w$ to $\partial \Omega$ and $C$ is a constant depending only on $\Omega$. They also asked if (1.1) could be improved to

$$
\operatorname{dist}_{\Omega}\left(w, w_{0}\right) \geq \frac{1}{C} \log \left(1 / \delta_{\Omega}(w)\right)
$$

which is known to be the best estimate for strongly pseudoconvex domains.

The main goal of this paper is to show that one can improve (1.1) to

$$
\operatorname{dist}_{\Omega}\left(w, w_{0}\right) \geq \frac{\log \left(1 / \delta_{\Omega}(w)\right)}{C \log \log \left(1 / \delta_{\Omega}(w)\right)}
$$

for $C^{2}$ smooth bounded pseudoconvex $\Omega$ in $\mathbb{C}^{n}$. Our main tool will be the pluricomplex Green function. We recall that for a bounded domain $\Omega$ in $\mathbb{C}^{n}$ and a pole $w \in \Omega$ it is defined by

$$
g_{\Omega, w}:=\sup \left\{u \in P S H(\Omega): u<0, \limsup _{z \rightarrow w}(u(z)-\log |z-w|)<\infty\right\} .
$$

We refer to [10] or 20 for basic properties of $g_{\Omega}$. The direct relation between the Bergman metric and the Green function has been explored quite extensively in recent years (see for example [6, 7, 13, 16]). In [14] a certain technical function similar but different from $g_{\Omega}$ was used. Here however, unlike in [14, we are able to apply the Green function directly. The main relation for us with the Bergman metric will be the following quite general result (it is a special case of Theorem 4.4 below).

Received by the editors May 29, 2003.

2000 Mathematics Subject Classification. Primary 32F45; Secondary 32U35.

This research was partially supported by KBN Grant \#2 P03A 02819.

(C)2005 American Mathematical Society Reverts to public domain 28 years from publication 
Theorem 1.1. There exists a positive constant $c_{n}$, depending only on $n$, such that if $\Omega$ is a bounded pseudoconvex domain in $\mathbb{C}^{n}$ and $w, \widetilde{w} \in \Omega$ are such that $\left\{g_{\Omega, w} \leq-1\right\} \cap\left\{g_{\Omega, \widetilde{w}}<-1\right\}=\emptyset$, then

$$
\operatorname{dist}_{\Omega}(w, \widetilde{w}) \geq c_{n} .
$$

The main ingredients of the proof of Theorem 1.1 are the Kobayashi lower bound for the Bergman distance [21] and an $L^{2}$-estimate for the $\bar{\partial}$ operator essentially due to Donnelly and Fefferman [15. As shown by Berndtsson [1, [3, it is in fact a simple consequence of the original Hörmander theory [18. It should be pointed out that in many papers (see for example [7], [8], [14]) much more complicated $L^{2}$-estimates for the $\bar{\partial}$ operator were used.

Therefore, thanks to Theorem 1.1, in order to get a lower bound for the Bergman distance, it is enough to estimate the pluricomplex Green function from below in order to study the behavior of its sublevel sets. We do it in Section 5 following two basic ideas due to Herbort [17] and Diederich-Herbort [13]. The first is to use an inequality for the complex Monge-Ampère operator from [4] to estimate $\left|g_{\Omega, w}(\widetilde{\zeta})\right|$ from above in terms of $\left|g_{\Omega, \zeta}(w)\right|$ for some $\widetilde{\zeta}$ close to $\zeta$. Then one estimates the modulus of continuity of $g_{\Omega, w}$ which is known to be continuous precisely when $\Omega$ is hyperconvex, that is, when it admits a bounded plurisubharmonic exhaustion function (see [10]). As a result, we improve some estimates from [17] and [13], by the way simplifying the part of Herbort's argument involving the estimate for the modulus of continuity of $g_{\Omega, w}$ [17, Main Lemma]). In particular, we get the following result (see Theorem 5.2 below with $a=b$ ).

Theorem 1.2. Let $\Omega$ be a bounded domain in $\mathbb{C}^{n}$ with diameter $R$ for which there exists $v \in P S H(\Omega)$ and positive constants $A$ and a such that in $\Omega$ we have

$$
\frac{1}{A} \delta_{\Omega}^{a} \leq|v| \leq A \delta_{\Omega}^{a}
$$

Then there exist positive constants $C_{1}, C_{2}$ depending only on $n, A, a$ and $R$ such that if $w \in \Omega$ is such that $r:=\delta_{\Omega}(w) \leq e^{-2}$, then

$$
\left\{g_{\Omega, w} \leq-1\right\} \subset\left\{C_{1}^{-1} r(\log (1 / r))^{-1 / a} \leq \delta_{\Omega} \leq C_{2} r(\log (1 / r))^{n / a}\right\} .
$$

We will now explain how Theorems 1.1 and 1.2 imply the estimate (1.3) for $C^{2}$ smooth pseudoconvex domains in $\mathbb{C}^{n}$. By [11] such domains satisfy the assumption of Theorem 1.2 and therefore (1.4) holds provided that

$$
\delta_{\Omega}(\widetilde{w}) \geq \delta_{\Omega}(w)\left(\log \left(1 / \delta_{\Omega}(w)\right)\right)^{C},
$$

where $C>1$ depends only on $\Omega$ and $\delta_{\Omega}(\widetilde{w}) \leq e^{-C}$. Assume that $r:=\delta_{\Omega}(w) \leq$ $\min \left\{e^{-C}, \delta_{\Omega}\left(w_{0}\right) / 2\right\}=: r_{0}$. The function $\gamma(\rho):=\rho(\log (1 / \rho))^{C}$ is increasing on the interval $\left(0, r_{0}\right]$. We can find an integer $k$ such that $\gamma^{k-1}(r) \leq r_{0} \leq \gamma^{k}(r)$, where $\gamma^{k}=\gamma \circ \cdots \circ \gamma$. Any curve joining $w$ with $w_{0}$ intersects the level sets $\left\{\delta_{\Omega}=\gamma^{j}(r)\right\}, j=1, \ldots, k-1$, and thus by (1.6) and Theorem 1.1 its Bergman length can be estimated from below by $(k-1) c_{n}$. One can show inductively that if $r(\log (1 / r))^{(k-1) C} \leq r_{0}$, then $\gamma^{k}(r) \leq r(\log (1 / r))^{k C}$. It follows that $r_{0} \leq$ $r(\log (1 / r))^{k C}$ which easily implies the estimate (1.3).

In 14 the jump in the Bergman distance was obtained for $w, \widetilde{w}$ with

$$
\delta_{\Omega}(\widetilde{w}) \geq \delta_{\Omega}(w)^{1 / C}
$$


and then (1.1) immediately followed. On the other hand, a slightly weaker condition than (1.5) was assumed in [14. To obtain (1.2) one would need to improve (1.6) to

$$
\delta_{\Omega}(\widetilde{w}) \geq C \delta_{\Omega}(w) .
$$

This we are able to prove in arbitrary bounded convex domains with the constant $C=(e+1)^{2} /(e-1)^{2}$ (Theorem 5.4 below). Thus, using the localization principle for the Bergman metric (see for example [12] or [22]), we obtain the following.

Theorem 1.3. Let $\Omega$ be a bounded domain in $\mathbb{C}^{n}$ such that for every $z_{0} \in \partial \Omega$ there exists an open neighborhood $U$ of $z_{0}$, open $V$ in $\mathbb{C}^{n}$ and a biholomorphism $F: U \rightarrow V$ such that $F(\Omega \cap U)$ is convex. Then there exists a positive constant $C$ depending only on $\Omega$ such that (1.2) holds for every $w_{0} \in \Omega$ and every $w \in \Omega$ sufficiently close to $\partial \Omega$.

Note that again no assumption is made on the regularity of $\Omega$ (of course in the latter case the boundary must always be Lipschitz continuous).

\section{The Kobayashi COnstruction}

In this section we will briefly sketch the construction of Kobayashi [21] and discuss some of its consequences. We assume that $\Omega$ is a bounded domain in $\mathbb{C}^{n}$. By $H^{2}(\Omega)$ we denote the Hilbert space of square integrable holomorphic functions in $\Omega$ and $K_{\Omega}(z, w)$ is the Bergman kernel of $\Omega$ (holomorphic in $z$, antiholomorphic in $w$ ). We define the immersion of $\Omega$ into the (infinitely dimensional) projective space $\mathbb{P}\left(H^{2}(\Omega)\right)$ as follows:

$$
\tau: \Omega \ni w \longmapsto\left[K_{\Omega}(\cdot, w)\right] \in \mathbb{P}\left(H^{2}(\Omega)\right) .
$$

One can show that the Bergman metric in $\Omega$ is precisely the pull-back of the FubiniStudy metric in $\mathbb{P}\left(H^{2}(\Omega)\right)$. Therefore

$$
\operatorname{dist}_{\Omega}(w, \widetilde{w}) \geq \operatorname{dist}_{\mathbb{P}\left(H^{2}(\Omega)\right)}(\tau(w), \tau(\widetilde{w})), \quad w, \widetilde{w} \in \Omega .
$$

Moreover, $\mathbb{P}\left(H^{2}(\Omega)\right)$ (with the Fubini-Study metric) is complete and

$$
\operatorname{dist}_{\mathbb{P}\left(H^{2}(\Omega)\right)}([f],[g])=\arccos \frac{|\langle f, g\rangle|}{\|f\|\|g\|}, \quad f, g \in H^{2}(\Omega) \backslash\{0\} .
$$

We can now easily deduce the following two results.

Proposition 2.1. For a bounded domain $\Omega$ in $\mathbb{C}^{n}$ we have

$$
\operatorname{dist}_{\Omega}(w, \widetilde{w}) \geq \arccos \frac{\left|K_{\Omega}(w, \widetilde{w})\right|}{\sqrt{K_{\Omega}(w, w) K_{\Omega}(\widetilde{w}, \widetilde{w})}}, \quad w, \widetilde{w} \in \Omega .
$$

Proposition 2.2. If a bounded domain $\Omega$ in $\mathbb{C}^{n}$ satisfies

$$
\limsup _{w \rightarrow \partial \Omega} \frac{|f(w)|}{\sqrt{K_{\Omega}(w, w)}}<\|f\|_{L^{2}(\Omega)}, \quad f \in H^{2}(\Omega) \backslash\{0\},
$$

then it is Bergman complete.

Proof. Let $w_{j}$ be a Cauchy sequence with respect to dist $\Omega$. Then let $\tau\left(w_{j}\right)$ be a Cauchy sequence with respect to $\operatorname{dist}_{\mathbb{P}\left(H^{2}(\Omega)\right)}$. Since $\mathbb{P}\left(H^{2}(\Omega)\right)$ is complete, we can find $f \in H^{2}(\Omega) \backslash\{0\}$ such that $\tau\left(w_{j}\right)=\left[K_{\Omega}\left(\cdot, w_{j}\right)\right] \rightarrow[f]$. In particular,

$$
\frac{\left|f\left(w_{j}\right)\right|}{\|f\| \sqrt{K_{\Omega}\left(w_{j}, w_{j}\right)}}=\left|\left\langle\frac{f}{\|f\|}, \frac{K_{\Omega}\left(\cdot, w_{j}\right)}{\left\|K_{\Omega}\left(\cdot, w_{j}\right)\right\|}\right\rangle\right| \rightarrow 1
$$


which by assumption means that $w_{j}$ has no accumulation point on $\partial \Omega$. But this of course means that $w_{j}$ is also a Cauchy sequence with respect to the euclidean metric.

Zwonek 24] constructed a bounded, Bergman complete domain in $\mathbb{C}$ not satisfying

$$
\limsup _{w \rightarrow \partial \Omega} \frac{|f(w)|}{\sqrt{K_{\Omega}(w, w)}}=0, \quad f \in H^{2}(\Omega)
$$

which was the criterion for Bergman completeness formulated in [21. It remains an open problem to construct a Bergman complete domain $\Omega$ in $\mathbb{C}^{n}$ such that the (possibly) weaker condition (2.1) does not hold.

Proposition 2.1 shows that in order to estimate $\operatorname{dist}_{\Omega}(w, \widetilde{w})$ from below we need to estimate $\left|K_{\Omega}(w, \widetilde{w})\right| / \sqrt{K_{\Omega}(w, w) K_{\Omega}(\widetilde{w}, \widetilde{w})}$ from above. Similarly as in [14, we will see that it is enough to construct a right function from $H^{2}(\Omega)$.

Proposition 2.3. Let $\Omega$ be a bounded domain in $\mathbb{C}^{n}, w, \widetilde{w} \in \Omega$. Suppose that $f \in H^{2}(\Omega)$ is such that $f(w)=K_{\Omega}(w, \widetilde{w}) / \sqrt{K_{\Omega}(\widetilde{w}, \widetilde{w})}$ and $f(\widetilde{w})=0$. Then

$$
\frac{\left|K_{\Omega}(w, \widetilde{w})\right|}{\sqrt{K_{\Omega}(w, w) K_{\Omega}(\widetilde{w}, \widetilde{w})}} \leq \frac{\|f\|_{L^{2}(\Omega)}}{\sqrt{1+\|f\|_{L^{2}(\Omega)}^{2}}}
$$

and

$$
\operatorname{dist}_{\Omega}(w, \widetilde{w}) \geq \frac{\pi}{2}-\arctan \|f\|_{L^{2}(\Omega)} .
$$

Proof. We first note that the second estimate is a direct consequence of the first one and Proposition 2.1. We may assume that $f \neq 0$. Set $h:=K_{\Omega}(\cdot, \widetilde{w}) / \sqrt{K_{\Omega}(\widetilde{w}, \widetilde{w})}$. Then $\langle f, h\rangle=f(\widetilde{w}) / \sqrt{K_{\Omega}(\widetilde{w}, \widetilde{w})}=0$ and therefore we can find an orthonormal basis $\left\{\varphi_{0}, \varphi_{1}, \ldots\right\}$ of $H^{2}(\Omega)$ such that $\varphi_{0}=h$ and $\varphi_{1}=f /\|f\|$. Then

$$
K_{\Omega}(z, z)=\sum_{j=0}^{\infty}\left|\varphi_{j}(z)\right|^{2} \geq|h(z)|^{2}+\frac{|f(z)|^{2}}{\|f\|^{2}}, \quad z \in \Omega .
$$

Applying it for $z=w$ we get the desired estimate.

\section{The Hörmander-Donnelly-FefFERMAn-BERndtsson $L^{2}$-ESTIMATE FOR THE $\bar{\partial}$ OPERATOR}

Our main tool in constructing square integrable holomorphic functions will be the following estimate for the $\bar{\partial}$ operator, essentially due to Donnelly and Fefferman [15].

Theorem 3.1. Let $\Omega$ be a pseudoconvex domain in $\mathbb{C}^{n}$ and let $\psi$ be a plurisubharmonic function in $\Omega$ such that $-e^{-\psi}$ is also plurisubharmonic. Assume that $\alpha \in L_{l o c,(0,1)}^{2}(\Omega)$ is such that $\bar{\partial} \alpha=0$ and that

$$
i \bar{\alpha} \wedge \alpha \leq H i \partial \bar{\partial} \psi
$$

for some nonnegative, locally integrable function $H$ in $\Omega$. Then for every plurisubharmonic $\varphi$ in $\Omega$ there exists $u \in L_{\text {loc }}^{2}(\Omega)$ with $\bar{\partial} u=\alpha$ and such that

$$
\int_{\Omega}|u|^{2} e^{-\varphi} \leq 16 \int_{\Omega} H e^{-\varphi} .
$$


Theorem 3.1 is a direct consequence of the next result (with $r=1 / 4$ and $\varphi, \psi$ replaced with $\varphi+r \psi, r \psi$, respectively), which is a slight improvement of an estimate due to Berndtsson [1, Theorem 3.1].

Theorem 3.2. Let $\Omega$ be a pseudoconvex domain in $\mathbb{C}^{n}$ and let $\psi$ be a plurisubharmonic function in $\Omega$ such that for some fixed $r \in(0,1)$, the function $-e^{-\psi / r}$ is also plurisubharmonic. Then for any $\alpha, H$ and $\varphi$ chosen as in Theorem 3.1 we can find $u \in L_{l o c}^{2}(\Omega)$ with $\bar{\partial} u=\alpha$ and

$$
\int_{\Omega}|u|^{2} e^{\psi-\varphi} \leq \frac{1}{(1-\sqrt{r})^{2}} \int_{\Omega} H e^{\psi-\varphi} .
$$

Proof. We first assume that $\Omega$ is smooth and $\varphi, \psi$ are smooth up to the boundary. We now follow the proof of [2, Lemma 2.2]. We have in particular $L^{2}(\Omega)=$ $L^{2}\left(\Omega, e^{a \varphi+b \psi}\right)$ for real $a, b$ and $-e^{-\psi / r} \in P S H(\Omega)$ means precisely that

$$
i \partial \psi \wedge \bar{\partial} \psi \leq r i \partial \bar{\partial} \psi
$$

Let $u$ be the solution to $\bar{\partial} u=\alpha$ which is minimal in the $L^{2}\left(\Omega, e^{-\varphi}\right)$ norm. This means that

$$
\int_{\Omega} u \bar{f} e^{-\varphi}=0, \quad f \in H^{2}(\Omega)
$$

Set $v:=e^{\psi} u$. Then

$$
\int_{\Omega} v \bar{f} e^{-\varphi-\psi}=0, \quad f \in H^{2}(\Omega),
$$

thus $v$ is the minimal solution in the $L^{2}\left(\Omega, e^{-\varphi-\psi}\right)$ norm to $\bar{\partial} v=\beta$, where

$$
\beta=\bar{\partial}\left(e^{\psi} u\right)=e^{\psi}(\alpha+u \bar{\partial} \psi) \text {. }
$$

For every $t>0$ we have

$$
\begin{aligned}
i \bar{\beta} \wedge \beta & \leq e^{2 \psi}\left[\left(1+t^{-1}\right) i \bar{\alpha} \wedge \alpha+(1+t)|u|^{2} i \partial \psi \wedge \bar{\partial} \psi\right] \\
& \leq e^{2 \psi}\left[\left(1+t^{-1}\right) H+(1+t) r|u|^{2}\right] i \partial \bar{\partial} \psi \\
& \leq e^{2 \psi}\left[\left(1+t^{-1}\right) H+(1+t) r|u|^{2}\right] i \partial \bar{\partial}(\varphi+\psi) .
\end{aligned}
$$

Therefore by [18, Lemma 4.4.1] (which is also true with the constant 2 replaced by 1) we get

$$
\int_{\Omega}|u|^{2} e^{\psi-\varphi}=\int_{\Omega}|v|^{2} e^{-\varphi-\psi} \leq\left(1+t^{-1}\right) \int_{\Omega} H e^{\psi-\varphi}+(1+t) r \int_{\Omega}|u|^{2} e^{\psi-\varphi} .
$$

For $t=r^{-1 / 2}-1$ we obtain the required result when $\varphi, \psi$ are smooth.

Now assume that $\Omega, \varphi$ are arbitrary and $\psi$ is strongly plurisubharmonic but otherwise arbitrary (possibly even not locally bounded). By the Radon-Nikodym theorem there exists $\beta=\sum_{j, k} \beta_{j k} i d z_{j} \wedge d \bar{z}_{k} \in L_{l o c,(1,1)}^{1}(\Omega)$ such that $0<\beta \leq i \partial \bar{\partial} \psi$ and $i \bar{\alpha} \wedge \alpha \leq H \beta$. For $\varepsilon>0$ let $\Omega_{\varepsilon} \subset \Omega$ be a smooth pseudoconvex domain such that $\varphi_{\varepsilon}:=\varphi * \rho_{\varepsilon}, \psi_{\varepsilon}:=\psi * \rho_{\varepsilon}$, the standard regularizations, are defined in a neighborhood of $\bar{\Omega}_{\varepsilon}$. If $\left(\psi_{\varepsilon}^{j k}\right)$ denotes the inverse matrix of $\left(\partial^{2} \psi_{\varepsilon} / \partial z_{j} \partial \bar{z}_{k}\right)$, then $H_{\varepsilon}:=\sum_{j, k} \psi_{\varepsilon}^{j k} \bar{\alpha}_{j} \alpha_{k}$ is the least function satisfying $i \bar{\alpha} \wedge \alpha \leq H_{\varepsilon} i \partial \bar{\partial} \psi_{\varepsilon}$. One can easily check that

$$
i \partial \psi_{\varepsilon} \wedge \bar{\partial} \psi_{\varepsilon} \leq(i \partial \psi \wedge \bar{\partial} \psi) * \rho_{\varepsilon} \leq r i \partial \bar{\partial} \psi_{\varepsilon}
$$


and thus $-e^{-\psi_{\varepsilon} / r}$ is also plurisubharmonic. By the previous part we can thus find $u_{\varepsilon} \in L_{l o c}^{2}\left(\Omega_{\varepsilon}\right)$ such that $\bar{\partial} u_{\varepsilon}=\alpha$ in $\Omega_{\varepsilon}$ and

$$
\int_{\Omega_{\varepsilon}}\left|u_{\varepsilon}\right|^{2} e^{\psi_{\varepsilon}-\varphi_{\varepsilon}} \leq \int_{\Omega_{\varepsilon}} H_{\varepsilon} e^{\psi_{\varepsilon}-\varphi_{\varepsilon}} \leq \int_{\Omega_{\varepsilon}} H_{\varepsilon} e^{\psi_{\varepsilon}-\varphi} .
$$

We have $\beta_{\varepsilon}:=\beta * \rho_{\varepsilon} \leq i \partial \bar{\partial} \psi_{\varepsilon}$ and there is a sequence $\varepsilon_{l} \downarrow 0$ such that the coefficients of $\beta_{\varepsilon_{l}}$ converge pointwise almost everywhere to the respective coefficients of $\beta$. Therefore

$$
\varlimsup_{l \rightarrow \infty} H_{\varepsilon_{l}} \leq \varlimsup_{l \rightarrow \infty} \sum_{j, k} \beta_{\varepsilon_{l}}^{j k} \bar{\alpha}_{j} \alpha_{k}=\sum_{j, k} \beta^{j k} \bar{\alpha}_{j} \alpha_{k} \leq H,
$$

where $\left(\beta^{j k}\right)$ and $\left(\beta_{\varepsilon}^{j k}\right)$ denote the inverse matrices of $\left(\beta_{j k}\right)$ and $\left(\beta_{j k} * \rho_{\varepsilon}\right)$, respectively. If we now assume that the left-hand side of (3.2) is equal to 1, by the Fatou lemma we have

$$
\varlimsup_{l \rightarrow \infty} \int_{\Omega_{\varepsilon_{l}}}\left|u_{\varepsilon_{l}}\right|^{2} e^{\psi_{\varepsilon_{l}}-\varphi_{\varepsilon_{l}}} \leq 1 .
$$

Since $\varphi_{\varepsilon_{l}}$ is a decreasing sequence and $\psi$ is locally bounded from above, we see that the $L^{2}$ norm of $u_{\varepsilon_{l}}$ over $\Omega_{\varepsilon}$ is bounded for every fixed $\varepsilon$. Therefore, replacing $\varepsilon_{l}$ with its subsequence if necessary, we see that $u_{\varepsilon_{l}}$ converges weakly in $\Omega_{\varepsilon}$ for every $\varepsilon$ to $u \in L_{l o c}^{2}(\Omega)$. We can now show that $u$ satisfies (3.2), which completes the proof for strongly plurisubharmonic $\psi$.

If $\psi$ is not necessarily strongly plurisubharmonic, then we may approximate it by functions of the form $\psi+\varepsilon|z|^{2}$. Note that $i \bar{\alpha} \wedge \alpha \leq H i \partial \bar{\partial}\left(\psi+\varepsilon|z|^{2}\right)$ and the general case easily follows along the same lines as before.

Note that the assumption on $\psi$ in Theorem 3.1 means precisely that $\psi$ is of the form

$$
\psi=-\log (-v),
$$

where $v$ is a negative plurisubharmonic function in $\Omega$. We shall usually use Theorem 3.1 for $\alpha$ given by

$$
\alpha=-f \bar{\partial}(\chi(\log (-v)),
$$

where $f$ is holomorphic and $\chi \in C^{0,1}(\mathbb{R})$. If $v$ is locally bounded near $\partial \Omega$, then by [9] $v \in W_{l o c}^{1,2}(\Omega)$, and thus $\alpha \in L_{(0,1), l o c}^{2}(\Omega)$. The inequality (3.1) is then satisfied with

$$
H=\left|f \chi^{\prime}(\log (-v))\right|^{2} .
$$

Theorem 3.1 thus gives the following result.

Theorem 3.3. Let $f$ be a holomorphic function in a pseudoconvex domain $\Omega$ in $\mathbb{C}^{n}$. Assume that $\varphi, v$ are plurisubharmonic in $\Omega$ such that $v<0$ and $v$ is locally bounded near $\partial \Omega$. Let moreover $\chi \in C^{0,1}(\mathbb{R})$. Then one can find a holomorphic function $F$ in $\Omega$ satisfying the following estimate:

$$
\int_{\Omega}|F-f \chi(\log (-v))|^{2} e^{-\varphi} \leq 16 \int_{\Omega}\left|f \chi^{\prime}(\log (-v))\right|^{2} e^{-\varphi}
$$




\section{Applichtions to the Bergman kernel And metric}

Theorems 3.1 and 3.3 seem to be quite universal tools in obtaining various estimates related to the Bergman kernel. First, we get the following estimate due to Herbort [16] (with a different constant though, depending also on the diameter of $\Omega)$.

Theorem 4.1. Let $\Omega$ be a bounded pseudoconvex domain in $\mathbb{C}^{n}$ and $f \in H^{2}(\Omega)$. Then

where

$$
\frac{|f(w)|}{\sqrt{K_{\Omega}(w, w)}} \leq\left(1+\frac{4}{\eta(n)}\right)\|f\|_{L^{2}\left(\left\{g_{\Omega, w} \leq-1\right\}\right)}, \quad w \in \Omega
$$

$$
\eta(y):=\int_{y}^{\infty} \frac{d x}{x e^{x}} .
$$

Proof. We apply Theorem 3.3 with $\varphi:=2 n g_{\Omega, w}, v:=g_{\Omega, w}$ and

$$
\chi(t):= \begin{cases}0, & t \leq 0, \\ \int_{0}^{t} e^{-n e^{s}} d s, & t>0 .\end{cases}
$$

We can find a holomorphic $F$ in $\Omega$ such that

$$
\begin{aligned}
\|F\|_{L^{2}(\Omega)} & \leq\|f \chi(\log (-v))\|_{L^{2}(\Omega)}+\|F-f \chi(\log (-v))\|_{L^{2}(\Omega)} \\
& \leq \chi(\infty)\|f\|_{L^{2}\left(\left\{g_{\Omega, w} \leq-1\right\}\right)}+\|F-f \chi(\log (-v))\|_{L^{2}\left(\Omega, e^{-\varphi}\right)} \\
& \leq \eta(n)\|f\|_{L^{2}\left(\left\{g_{\Omega, w} \leq-1\right\}\right)}+4\left\|f \chi^{\prime}(\log (-v))\right\|_{L^{2}\left(\Omega, e^{-\varphi}\right)} \\
& \leq(\eta(n)+4)\|f\|_{L^{2}\left(\left\{g_{\Omega, w} \leq-1\right\}\right) .}
\end{aligned}
$$

Since $e^{-\varphi}$ is not integrable near $w$, from Theorem 3.3 it also follows that $F(w)=$ $\chi(\infty) f(w)=\eta(n) f(w)$. Hence

$$
\frac{|f(w)|}{\sqrt{K_{\Omega}(w, w)}}=\frac{|F(w)|}{\eta(n) \sqrt{K_{\Omega}(w, w)}} \leq \frac{\|F\|_{L^{2}(\Omega)}}{\eta(n)} .
$$

Theorem 3.3 together implies in particular, thanks to Proposition 2.2, that if $\Omega$ is bounded pseudoconvex in $\mathbb{C}^{n}$ and

then $\Omega$ must be Bergman complete.

$$
\lim _{w \rightarrow \partial \Omega} \operatorname{vol}\left(\left\{g_{\Omega, w} \leq-1\right\}\right)=0,
$$

Next, we generalize results of Chen 8] to several variables.

Theorem 4.2. Let $\Omega$ and $U$ be bounded domains in $\mathbb{C}^{n}$ such that $\Omega \cup U$ is pseudoconvex with diameter $R$. Assume that $U \subset B\left(z_{0}, r\right)$. Then for every $f \in H^{2}(\Omega)$ there exists $F \in H^{2}(\Omega \cup U)$ such that for every $\lambda>1$ we have

$$
\|F-f\|_{L^{2}(\Omega)} \leq\left(1+\frac{4}{\log \lambda}\right)\|f\|_{L^{2}\left(\Omega \cap \bar{B}\left(z_{0},(r / R)^{1 / \lambda}\right)\right)} .
$$

Proof. We set $v(z):=\log \left|z-z_{0}\right| / R, \varphi:=0$ and, for $\rho>r$,

$$
\chi(t):= \begin{cases}1, & t \leq \log \log (R / \rho)-\log \lambda, \\ \frac{\log \log (R / \rho)-t}{\log \lambda}, & \log \log (R / \rho)-\log \lambda<t \leq \log \log (R / \rho), \\ 0, & t>\log \log (R / \rho) .\end{cases}
$$


Note that (3.3) defines $\bar{\partial}$-closed $\alpha \in L_{(0,1), l o c}^{2}(\Omega \cup U)$. We obtain a holomorphic $F$ with

$$
\|F-f\|_{L^{2}(\Omega)} \leq\|f(1-\chi(\log (-v)))\|_{L^{2}(\Omega)}+4|| f \chi^{\prime}(\log (-v)) \|_{L^{2}(\Omega)}
$$

and the desired estimate will follow if we let $\rho$ tend to $r$.

Corollary 4.3. Assume that $\Omega$ is a bounded domain in $\mathbb{C}^{n}$ satisfying the following property: for every $z_{0} \in \partial \Omega$ there exists a neighborhood basis $U_{j}$ of $z_{0}$ such that $\Omega \cup U_{j}$ is pseudoconvex for every $j$. Then, if

$$
\lim _{w \rightarrow \partial \Omega} K_{\Omega}(w, w)=\infty
$$

it follows that $\Omega$ is Bergman complete.

Proof. Let $\Omega \ni w_{k} \rightarrow z_{0} \in \partial \Omega$ and $f \in H^{2}(\Omega)$. By Theorem 4.2 there exists a sequence $F_{j} \in H^{2}\left(\Omega \cup U_{j}\right)$ such that $\left\|F_{j}-f\right\|_{L^{2}(\Omega)} \rightarrow 0$. We have

$$
\frac{\left|f\left(w_{k}\right)\right|}{\sqrt{K_{\Omega}\left(w_{k}, w_{k}\right)}} \leq \frac{\left|F_{j}\left(w_{k}\right)\right|}{\sqrt{K_{\Omega}\left(w_{k}, w_{k}\right)}}+\left\|F_{j}-f\right\|_{L^{2}(\Omega)} .
$$

For every fixed $j$, since $F_{j}$ is holomorphic in a neighborhood of $z_{0}$, the sequence $\left|F_{j}\left(w_{k}\right)\right|$ is bounded. Thus, if we first let $k \rightarrow \infty$ and then $j \rightarrow \infty$, the corollary follows from Proposition 2.2.

Note that the assumption on $\Omega$ in Corollary 4.3 is always true if $n=1$ but not if $n>1$ : it is not satisfied for the Hartogs triangle $\Omega=\left\{(z, w) \in \mathbb{C}^{2}:|z|<|w|<1\right\}$.

Theorem 4.4. Let $\Omega$ be a bounded pseudoconvex domain in $\mathbb{C}^{n}$. Assume that $w, \widetilde{w} \in \Omega$ and $\alpha, \widetilde{\alpha}>0$ are such that $\left\{g_{\Omega, w} \leq-\alpha\right\} \cap\left\{g_{\Omega, \tilde{w}}<-\widetilde{\alpha}\right\}=\emptyset$. Then

$$
\operatorname{dist}_{\Omega}(w, \widetilde{w}) \geq \frac{\pi}{2}-\arctan \left(1+4 \frac{e^{n \widetilde{\alpha}}}{\eta(n \alpha)}\right)
$$

where $\eta$ is as in Theorem 4.1 .

Proof. Let $h:=K_{\Omega}(\cdot, \widetilde{w}) / \sqrt{K_{\Omega}(\widetilde{w}, \widetilde{w})} \in H^{2}(\Omega)$ so that $\|h\|_{L^{2}(\Omega)}=1$. Set $\varphi:=$ $2 n\left(g_{\Omega, w}+g_{\Omega, \tilde{w}}\right), v:=g_{\Omega, w}$ and

$$
\chi(t):= \begin{cases}0, & t \leq \log \alpha, \\ \int_{\log \alpha}^{t} e^{-n e^{s}} d s, & t>\log \alpha .\end{cases}
$$

Then in particular $\chi(\log (-v))=\chi(\infty)=\eta(n \alpha)$ at $w$ and $\chi(\log (-v))=0$ at $\widetilde{w}$. By Theorem 3.3 there exists $f \in H^{2}(\Omega)$ such that

$$
\begin{aligned}
\|f\|_{L^{2}(\Omega)} & \leq\|h \chi(\log (-v))\|_{L^{2}(\Omega)}+\|f-h \chi(\log (-v))\|_{L^{2}(\Omega)} \\
& \leq 1+\|f-h \chi(\log (-v))\|_{L^{2}\left(\Omega, e^{-\varphi}\right)} \\
& \leq 1+4\left\|h \chi^{\prime}(\log (-v))\right\|_{L^{2}\left(\Omega, e^{-\varphi}\right)} \\
& \leq 1+4 \frac{e^{n \widetilde{\alpha}}}{\eta(n \alpha)} .
\end{aligned}
$$

Since $e^{-\varphi}$ is not integrable near $w$ and $\widetilde{w}$, it also follows that $f(w)=h(w)$ and $f(\widetilde{w})=0$. It is now sufficient to apply Proposition 2.3. 


\section{Estimates For the Pluricomplex Green function}

The following theorem will be the main step in estimating the Green function. The main idea of the proof comes from [17] (see also [13]).

Theorem 5.1. Assume that $\Omega$ is a bounded hyperconvex domain in $\mathbb{C}^{n}$ with the diameter $R$. Let $\zeta, w \in \Omega$ and $0<\varepsilon<\min \{r / 2,|\zeta-w| / 2\}$, where $r:=\delta_{\Omega}(w)$. Then

$$
\left|g_{\Omega, w}(\zeta)\right| \leq \frac{\log (R / \varepsilon)}{\log (r /(2 \varepsilon))}\left(\sup _{\left\{\delta_{\Omega}=\varepsilon\right\}}\left|g_{\Omega, w}\right|+(n !)^{1 / n}(\log (R / \varepsilon))^{1-1 / n}\left|g_{\Omega, \zeta}(w)\right|^{1 / n}\right) .
$$

Proof. By [10] (see also 20]) the function $g_{\Omega, w}$ is continuous on $\bar{\Omega} \backslash\{w\}$ (with $g_{\Omega, w}=0$ on $\left.\partial \Omega\right)$. Let $\alpha:=\log (R / \varepsilon)$. By [4] and since $\left(d d^{c} g_{\Omega, w}\right)^{n}=(2 \pi)^{n} \delta_{w}$,

$$
\int_{\Omega}\left|g_{\Omega, w}\right|^{n}\left(d d^{c} \max \left\{g_{\Omega, \zeta},-\alpha\right\}\right)^{n} \leq n !(2 \pi)^{n} \alpha^{n-1}\left|g_{\Omega, \zeta}(w)\right| .
$$

The measure $\left(d d^{c} \max \left\{g_{\Omega, \zeta},-\alpha\right\}\right)^{n}$ is supported on the set $\left\{g_{\Omega, \zeta}=-\alpha\right\} \subset \bar{B}(\zeta, \varepsilon)$ and its total mass is equal to $(2 \pi)^{n}$. Therefore, there exists $\widetilde{\zeta} \in \bar{B}(\zeta, \varepsilon)$ such that

$$
\left|g_{\Omega, w}(\widetilde{\zeta})\right|^{n} \leq n !(\log (R / \varepsilon))^{n-1}\left|g_{\Omega, \zeta}(w)\right| .
$$

By $u$ denote the relative extremal function of the ball $\bar{B}(w, \varepsilon)$, that is,

$$
u=\sup \left\{v \in P S H(\Omega):\left.v\right|_{\Omega}<0,\left.v\right|_{\bar{B}(w, \varepsilon)} \leq-1\right\} .
$$

By [23] (see also [5]) the function $u$ is continuous on $\bar{\Omega}$ (with $u=0$ on $\partial \Omega$ and $u=-1$ on $\bar{B}(w, \varepsilon))$. One can easily check that

$$
\log (R / \varepsilon) u \leq g_{\Omega, w} \leq \log (r / \varepsilon) u \quad \text { on } \Omega \backslash B(w, \varepsilon)
$$

(since the functions are maximal in $\Omega \backslash \bar{B}(w, \varepsilon)$ and vanish on $\partial \Omega$, it is enough to show these inequalities on $\partial B(w, \varepsilon))$. In particular,

$$
|u(z)| \leq \frac{\sup _{\left\{\delta_{\Omega}=\varepsilon\right\}}\left|g_{\Omega, w}\right|}{\log (r / \varepsilon)}=: \delta, \quad \text { if } \delta_{\Omega}(z) \leq \varepsilon
$$

Set $\widetilde{\Omega}:=\{z \in \Omega: z+\widetilde{\zeta}-\zeta \in \Omega\}$ and for $\delta^{\prime}>\delta$

$$
h(z):= \begin{cases}\max \left\{u(z), u(z+\widetilde{\zeta}-\zeta)-\delta^{\prime}\right\}, & z \in \widetilde{\Omega}, \\ u(z), & z \in \Omega \backslash \widetilde{\Omega} .\end{cases}
$$

We claim that $h$ is a negative plurisubharmonic function in $\Omega$. Indeed, for $z \in \Omega \cap \partial \widetilde{\Omega}$ we have $\delta_{\Omega}(z) \leq \varepsilon$ and $u(z) \geq-\delta>u(z+\widetilde{\zeta}-\zeta)-\delta^{\prime}$, hence $h=u$ in a neighborhood of $\Omega \cap \partial \widetilde{\Omega}$ and it follows that $h$ is plurisubharmonic. We have

$$
u(z) \leq \frac{\log (|z-w| / r)}{\log (r / \varepsilon)}, \quad z \in \Omega \backslash B(w, \varepsilon)
$$

(because both functions are maximal and the inequality holds on the boundary), and

Therefore

$$
z+\widetilde{\zeta}-\zeta \in \bar{B}(w, 2 \varepsilon) \subset B(w, r), \quad z \in \bar{B}(w, \varepsilon) \subset \widetilde{\Omega} .
$$

and

$$
u(z+\widetilde{\zeta}-\zeta) \leq-\beta, \quad z \in \bar{B}(w, \varepsilon),
$$

$$
h \leq \max \left\{-1,-\beta-\delta^{\prime}\right\} \leq-\beta \text { on } \bar{B}(w, \varepsilon),
$$


where

$$
\beta:=\frac{\log (r /(2 \varepsilon))}{\log (r / \varepsilon)} .
$$

From the definition of $u$ it thus follows that $h(z) / \beta \leq u(z)$ for $z \in \Omega$. For $z=\zeta \in \widetilde{\Omega}$ letting $\delta^{\prime}$ tend to $\delta$ we get

$$
u(\widetilde{\zeta})-\delta \leq h(\zeta) \leq \beta u(\zeta),
$$

which together with (5.1) and (5.2) gives the required inequality.

Remark. For $n=1$ the theorem recovers the symmetry of $g_{\Omega}$ : it is enough to let $\varepsilon \rightarrow 0$.

Theorem 5.2. Let $\Omega$ be a bounded domain in $\mathbb{C}^{n}$, where we can find $v \in P S H(\Omega)$ and positive constants $A, B, a, b$ such that in $\Omega$ the following estimate holds:

$$
\frac{1}{A} \delta_{\Omega}^{a} \leq|v| \leq B \delta_{\Omega}^{b} .
$$

Then there exist positive constants $C, \widetilde{C}$ depending only on $n, A, B, a, b$ and $R$, the diameter of $\Omega$ such that for $\zeta, w \in \Omega$ with $r:=\delta_{\Omega}(w) \leq e^{-2}$ and $\rho:=\delta_{\Omega}(\zeta) \leq e^{-2}$ we have

$$
\left|g_{\Omega, w}(\zeta)\right| \leq \begin{cases}C \frac{\rho^{b}}{r^{a}} \log (1 / r), & \text { if } \rho \leq r / 2, \\ \widetilde{C} \frac{r^{b / n}}{\rho^{a / n}}(\log (1 / r))^{1-1 / n}(\log (1 / \rho))^{1 / n}, & \text { if } \rho \geq 2 r .\end{cases}
$$

In particular,

$$
\left\{g_{\Omega, w} \leq-1\right\} \subset\left\{C^{-1 / b} r^{a / b}(\log (1 / r))^{-1 / b} \leq \delta_{\Omega} \leq \widetilde{C}^{n / a} r^{b / a}(\log (1 / r))^{n / a}\right\} .
$$

Proof. Assume first that $\rho \leq r / 2$. We have

$$
g_{\Omega, w}(z) \geq \log (|z-w| / R), \quad z \in \Omega .
$$

Therefore

$$
g_{\Omega, w} \geq \frac{\log (2 R / r)}{\inf _{\bar{B}(w, r / 2)}|v|} v \quad \text { in } \Omega \backslash B(w, r / 2)
$$

(because the inequality holds on the boundary) and (5.3) now gives

$$
\left|g_{\Omega, w}(\zeta)\right| \leq 2^{a} A B \frac{\rho^{b}}{r^{a}} \log (2 R / r)
$$

which implies the first inequality in (5.4).

Now assume that $\rho \geq 2 r$. By $C_{1}, C_{2}, \ldots$ we will denote positive constants depending only on $n, A, B, a, b$ and $R$. If $0<\varepsilon<r / 2$, then by the first inequality

$$
\sup _{\left\{\delta_{\Omega}=\varepsilon\right\}}\left|g_{\Omega, w}\right| \leq C_{1} \frac{\varepsilon^{b}}{r^{a}} \log (1 / r)
$$

and

$$
\left|g_{\Omega, \zeta}(w)\right| \leq C_{1} \frac{r^{b}}{\rho^{a}} \log (1 / \rho),
$$

if $r$ is sufficiently small. Therefore, by Theorem 5.1

$$
\left|g_{\Omega, w}(\zeta)\right| \leq C_{2} \frac{\log (1 / \varepsilon)}{\log (r /(2 \varepsilon))}\left(\frac{\varepsilon^{b}}{r^{a}} \log (1 / r)+\frac{r^{b / n}}{\rho^{a / n}}(\log (1 / \varepsilon))^{1-1 / n}(\log (1 / \rho))^{1 / n}\right) .
$$


We set

$$
\varepsilon:=r^{\alpha}(\log (1 / r))^{-\frac{n-1}{b n}},
$$

where

since $a \geq b$. Then

$$
\alpha:=\frac{a(n-1)}{b n}+\frac{1}{n}+1 \geq 2,
$$

$$
\begin{aligned}
\frac{\varepsilon^{b}}{r^{a}} \log (1 / r) & \leq r^{(b-a) / n}(\log (1 / r))^{1 / n} \\
& \leq r^{(b-a) / n}(\log (1 / \varepsilon))^{1-1 / n}(\log (1 / r))^{1 / n} \\
& \leq C_{3} \frac{r^{b / n}}{\rho^{a / n}}(\log (1 / \varepsilon))^{1-1 / n}(\log (1 / \rho))^{1 / n}
\end{aligned}
$$

(recall that $\rho \geq 2 r$ ). We also have

$$
\frac{\log (1 / \varepsilon)}{\log (r /(2 \varepsilon))} \leq C_{4}
$$

and

$$
\log (1 / \varepsilon) \leq C_{5} \log (1 / r)
$$

Combining (5.5)-(5.8) we arrive at the second inequality in (5.4).

Theorem 5.2 immediately gives the following result which slightly generalizes the main result from [17.

Corollary 5.3. Let $\Omega$ be as in Theorem 5.2. Then for every compact subset $K$ of $\Omega$ we have

$$
\lim _{w \rightarrow \partial \Omega} \sup _{K}\left|g_{\Omega, w}\right|=0 .
$$

Remark. It remains an open problem if Corollary 5.3 holds for arbitrary bounded hyperconvex $\Omega$. Note that then we know from [6] that

$$
\lim _{w \rightarrow \partial \Omega}\left\|g_{\Omega, w}\right\|_{L^{p}(\Omega)}=0
$$

for every $p<\infty$.

Theorem 5.2 can be improved and its proof simplified when $\Omega$ is convex.

Theorem 5.4. Let $\Omega$ be a bounded convex domain in $\mathbb{C}^{n}$. For given $\zeta, w \in \Omega$ set $\rho:=\delta_{\Omega}(\zeta), r:=\delta_{\Omega}(w)$. Then

In particular,

$$
g_{\Omega, w}(\zeta) \geq \log \frac{|\rho-r|}{\rho+r} .
$$

$$
\left\{g_{\Omega, w} \leq-1\right\} \subset\left\{\frac{e-1}{e+1} r \leq \delta_{\Omega} \leq \frac{e+1}{e-1} r\right\} .
$$

Proof. By the Lempert theorem (see [19]) $g_{\Omega, w}(\zeta)$ is symmetric in $w$ and $\zeta$ and thus we way assume that $\rho>r$. Let $H$ be a real hyperplane in $\mathbb{C}^{n}$ with $H \cap \Omega=\emptyset$ and $\delta_{\Omega}(w)=\operatorname{dist}(w, H)$. After an orthonormal change of variables we may assume that $H=\left\{\operatorname{Re} z_{1}=0\right\}, \Omega \subset\left\{\operatorname{Re} z_{1}>0\right\}, w=(r, 0, \ldots, 0)$ and $\widetilde{\rho}:=\operatorname{dist}(\zeta, H)=$ $\operatorname{Re} \zeta_{1} \geq \rho$. Then

$$
g_{\Omega, w}(\zeta) \geq \log \frac{\left|\zeta_{1}-r\right|}{\left|\zeta_{1}+r\right|} \geq \log \frac{\widetilde{\rho}-r}{\widetilde{\rho}+r} \geq \log \frac{\rho-r}{\rho+r} .
$$


Remark. Actually, one can avoid the use of the Lempert theorem in the proof of Theorem 5.4. Namely, for $\rho<r$ one has to repeat the same argument but with the hyperplane $\widetilde{H}$ such that $\widetilde{H} \cap \Omega=\emptyset$ and $\delta_{\Omega}(\zeta)=\operatorname{dist}(\zeta, \widetilde{H})$.

\section{ACKNOWLEDGEMENTS}

This paper was written during the author's stay at the Max Planck Institute for Mathematics in the Sciences in Leipzig. He would like to express his gratitude for hospitality, in particular to Professor J. Jost.

\section{REFERENCES}

[1] B. Berndtsson, The extension theorem of Ohsawa-Takegoshi and the theorem of DonnellyFefferman, Ann. Inst. Fourier 46 (1996), 1083-1094. MR.1415958 (97k:32019)

[2] B. Berndtsson, Weighted estimates for the $\bar{\partial}$-equation, Complex Analysis and Geometry, Columbus, Ohio, 1999, Ohio State Univ. Math. Res. Inst. Publ., vol. 9, Walter de Gruyter, 2001, pp. 43-57. MR912730 (2003f:32049)

[3] B. Berndtsson, P. Charpentier, A Sobolev mapping property of the Bergman kernel, Math. Z. 235 (2000), 1-10. MR1785069 (2002a:32039)

[4] Z. Błocki, Estimates for the complex Monge-Ampère operator, Bull. Pol. Acad. Sci. 41 (1993), 151-157. MF $1414762(97 \mathrm{j}: 32009)$

[5] Z. Błocki, The complex Monge-Ampère operator in hyperconvex domains, Ann. Scuola Norm. Sup. Pisa 23 (1996), 721-747.

[6] Z. Błocki, P. Pflug, Hyperconvexity and Bergman completeness, Nagoya Math. J. 151 (1998), 221-225. MF 1469572 (98j:32009)

[7] B.-Y.Chen, Completeness of the Bergman metric on non-smooth pseudoconvex domains, Ann. Pol. Math. 71 (1999), 241-251. MR1704301 (2000i:32021)

[8] B.-Y.Chen, A remark on the Bergman completeness, Complex Variables Theory Appl. 42 (2000), 11-15. MR1786123 (2001e:32049)

[9] J.-P. Demailly, Mesures de Monge-Ampère et caractérisation géométrique des variétés algébraiques affines, Mémoire Soc. Math. de France 19 (1987).

[10] J.-P.Demailly, Mesures de Monge-Ampère et mesures plurisousharmoniques, Math.Z. 194 (1987), 519-564. MR0881709 (88g:32034)

[11] K.Diederich, J.E. Fornæss, Pseudoconvex domains: Bounded plurisubharmonic exhaustion functions, Invent. Math. 39 (1977), 129-141. MR0437806 (55:10728)

[12] K. Diederich, J.E. Fornæss, G. Herbort, The boundary behavior of the Bergman metric, Proc. Symp. Pure Math. 41 (1984), 59-67. MF0740872 (85j:32039)

[13] K. Diederich, G. Herbort, Quantitative estimates for the Green function and an application to the Bergman metric, Ann. Inst. Fourier 50 (2000), 1205-1228. MR.1799743 (2001k:32058)

[14] K. Diederich, T. Ohsawa, An estimate for the Bergman distance on pseudoconvex domains, Ann. of Math. 141 (1995), 181-190. MR,1314035 (95j:32039)

[15] H. Donnelly, C. Fefferman, $L^{2}$-cohomology and index theorem for the Bergman metric, Ann. of Math. 118 (1983), 593-618. MR0727705 (85f:32029)

[16] G. Herbort, The Bergman metric on hyperconvex domains, Math.Z. 232 (1999), 183-196. MR.1714284 (2000i:32020)

[17] G. Herbort, The pluricomplex Green function on pseudoconvex domains with a smooth boundary, Internat. J. Math. 11 (2000), 509-522. MR,1768171 (2001e:32051)

[18] L. Hörmander, An introduction to complex analysis in several variables, D. van Nostrand, Princeton, 1966. MR0203075 (34:2933)

[19] M. Jarnicki, P. Pflug, Invariant distances and metrics in complex analysis, Walter de Gruyter, 1993. MR $1242120(94 \mathrm{k}: 32039)$

[20] M. Klimek, Pluripotential Theory, Clarendon Press, 1991. MR1150978 (93h:32021)

[21] S. Kobayashi, Geometry of bounded domains, Trans. Amer. Math. Soc. 92 (1959), 267-290. MR 0112162(22:3017)

[22] J. McNeal, Lower bounds on the Bergman metric near a point of finite type, Ann. of Math. 136 (1992), 339-360. MR1185122 (93k:32051) 
[23] J.B. Walsh, Continuity of envelopes of plurisubharmonic functions, J. Math. Mech. 18 (1968), 143-148. MF 0227465 (37:3049)

[24] W.Zwonek, An example concerning Bergman completeness, Nagoya Math. J. 164 (2001), 89-101. MR.1869096 (2002i:32010)

Jagiellonian University, Institute of Mathematics, Reymonta 4, 30-059 Kraków, Poland - And - MaX-Planck-Institute for Mathematics in the Sciences, Inselstr. 22-26, 04103 Leipzig, Germany

E-mail address: blocki@im.uj.edu.pl 